\title{
Systemisches Arbeiten inder
}

\section{Heilpädagogischen}

Förderung mit dem Pferd

\section{Theoretische Grundlagen}

\section{Hildegard Stockhausen}

Der Beitrag beschäftigt sich mit Grundlagen des systemischen Denkens und deren Integration in die Heilpädagogische Förderung mit dem Pferd. Einige Formen der Arbeit mit dem Pferd im systemischen Kontext wie die Einzelförderung mit dem Kind und die Arbeit mit Kind und Bezugspersonen am Pferd werden vorgestellt.

\section{$\rightarrow$ Schlüsselbegriffe:}

Systemische Perspektive, Heilpädagogische Förderung mit dem Pferd, Beziehungsmuster, Ressourcenorientierung, Joining, Therapeutisches Reiten 
Liebe Leserinnen und Leser!

Hier finden Sie den in der Zeitschrift „Mensch und Pferd international“ Heft 2/2010 angekündigten ersten Teil des Fachbeitrags von Hildegard Stockhausen, der die theoretischen Grundlagen der systemischen Arbeit mit dem Pferd umfasst. Dieser Teil, der das Fallbeispiel untermauert, verdeutlicht, welche besonderen Eigenschaften das Pferd besonders geeignet für den Einsatz in der systemischen Arbeit machen, und gibt einen kurzen Überblick über die möglichen Formen der systemischen Arbeit mit dem Pferd.

In der pädagogischen Praxis hat in den vergangenen Jahren die systemische Sichtweise auffälligen Verhaltensweisen und Problemen gegenüber zunehmend an Bedeutung gewonnen. Der systemische Ansatz sieht kindliches Verhalten und dessen Entwicklung in einem engen Zusammenhang mit den sozialen Beziehungsstrukturen, in denen sich das Kind ${ }^{1}$ befindet, wie Familie, Kindergarten und Schule. Auffälligkeiten sind aus dieser Perspektive daher keine Eigenschaften des Kindes, sondern funktionale Bestandteile dieser Systeme und deren Beziehungsmuster. Eine solche Sichtweise setzt demnach auch den Einbezug der sozialen Kontexte des Kindes in der pädagogischen Arbeit voraus (Balgo/Voß 1995, 169).

Auch im Therapeutischen Reiten hat sich der Blickwinkel vorgestellten Problemen und familiären Wirklichkeiten gegenüber geändert und die pädagogische Handlungsweise beeinflusst. Im Folgenden wird beschrieben, wie über das Pferd sowohl in der Beziehungsarbeit mit den Kindern als auch mit den Familien systemisch gearbeitet werden kann. Das Pferd ist für alle Beteiligten meist neutral besetzt und bietet durch seine artspezifischen Eigenschaften und sein Lebens- umfeld allein schon „einen Unterschied, der einen Unterschied macht“ (Simon 1993).

\section{Formen systemischer Familienarbeit mit dem}

Pferd An dieser Stelle sollen neben dem Einstieg in die Arbeit mit dem Pferd im systemischen Kontext nur zwei mögliche Settings kurz vorgestellt werden: die Arbeit mit dem Kind am Pferd in der Einzelförderung und die Arbeit mit Kind und Eltern ${ }^{2}$ gemeinsam am Pferd.

\section{Erstkontakt, Vorstellung und Anmeldung}

Der erste informelle Kontakt erfolgt in der Regel telefonisch auf Initiative der Eltern hin. Mir dient dieser Erstkontakt dazu, neben Informationen über die Familie, den Überweisungskontext und den Auftrag der Eltern an mich zu erfragen. Ferner bilde ich aus den bisherigen Darstellungen bereits erste Hypothesen, die im weiteren Verlauf der Arbeit immer wieder auf ihre Richtigkeit und Nützlichkeit hin überprüft und verändert werden. Bei der Bildung von Hypothesen geht es um die Erweiterung von Perspektiven und Möglichkeiten sowohl für den Therapeuten als auch für die Familie.

Bei Interesse an einer Therapieteilnahme wird ein unverbindlicher Informationstermin zur Vorstellung des Kindes und der Familie auf dem Pferdehof vereinbart, der zeitlich meist zwischen zwei Therapieeinheiten oder im Anschluss daran liegt, so dass die Eltern und das Kind die Möglichkeit haben, einen kurzen Einblick in meine Arbeit zu erhalten, die örtlichen Gegebenheiten allein zu erkunden, sich mit wartenden Eltern auszutauschen und die Pferde kennen zu lernen. Diese Faktoren wirken sowohl bei den Eltern als auch bei den meist unter Anforderungsdruck stehenden Kindern angstreduzierend und entspannend und bilden auf diese Weise ein optimales „Joining“ für den Beginn einer guten Zusammenarbeit.

1 Der Einfachheit halber ist nachfolgend die Rede von Kindern, die Arbeit mit Jugendlichen ist jedoch ebenso gemeint.

2 Im Sinne einer besseren Lesbarkeit wird im Folgenden von Eltern gesprochen. Die Elternarbeit bezieht sich natürlich ebenfalls auf Bezugspersonen aus Kinderheim, Wohngruppe und Pflegefamilie. 
Als nächster Schritt erfolgt die Kontaktaufnahme des Kindes zu mir und zum Pferd, bei der ich neben einem ersten Einblick in dessen motorische und kognitive Fähigkeiten ebenfalls Informationen über die Eltern-Kind-Interaktion erhalte. Im anschließenden Gespräch mit der Familie stelle ich ihnen kurz mein systemisch-pädagogisches Konzept vor, kläre die Rahmenbedingungen und die Voraussetzungen für den Beginn einer Teilnahme, wie die Bereitschaft der Eltern an einer aktiven Elternarbeit.

Kommt es zu einer Anmeldung, erhalten die Eltern zusammen mit den Anmeldeunterlagen einen standardisierten „Orientierungsbogen“, der einen groben Überblick über die bisherige Entwicklung des Kindes geben und das Problem aus Sicht der Anmeldenden kurz darstellen soll (Details dazu in: Stockhausen 2009, 17).

\section{Systemisches Arbeiten mit dem Kind am Pferd}

Den Einstieg in die systemische Arbeit mit dem Kind am Pferd bildet das Ergründen der eigenen Emotionen zu Beginn einer Fördereinheit, das über das Lebewesen Pferd beispielsweise in Form einer vergleichenden Beobachtung der aktuellen Gefühlslage des Pferdes und der eigenen Person erfolgt (z.B. „Wer ist - auf einer Skala von eins bis zehn - heute besser gelaunt/wacher / müder, das Pferd oder du?"). Alternativ dazu kann dieser Prozess über den Vergleich von Pferden in der Gruppe erfolgen, bei dem sich die Kinder aus der Herde ein Pferd aussuchen, das ihrer heutigen Stimmung am besten entspricht, und eins, das die Verfassung verkörpert, die das Kind am Ende der Stunde, eines Monats, der gesamten Maßnahme usw. gerne hätte. Die vom Kind durch beide Vorgehensweisen angesprochenen Bedürfnisse werden vom Pädagogen aufgegriffen und in der Arbeit mit dem Pferd umgesetzt, ebenso wie die zum Teil konträre Veränderung der jeweiligen Bedürfnisse im Verlauf der Einheit (Details dazu in: Stockhausen 2009, 18f).

Das systemische Arbeiten mit dem Kind am Pferd bietet ebenfalls die Möglichkeit, Infor- mationen über das Familiensystem ohne deren physische Anwesenheit zu erhalten und meine Hypothesen zu deren Beziehungsmustern in die Arbeit mit dem Kind zu integrieren. So können z. B. nach erfolgreicher Bewältigung einer schwierigen Übung systemische Fragen an das Kind meine Hypothesen auf ihre Eignung hin überprüfen, z.B. "Was glaubst Du, würde Dein Vater sagen, wenn er Dich bei dieser Aufgabe sehen würde?" oder: „Welches Mitglied der Familie würdest Du gerne mit aufs Pferd nehmen, welches an zweiter Stelle, wen auf gar keinen Fall?“. Die hier getroffenen Aussagen machen die Beziehungen und Strukturen innerhalb der Familie deutlich. Meine Beobachtungen hierzu werden in der weiteren $\mathrm{Ar}$ beit mit dem Kind und - mit seinem Einverständnis - auch innerhalb der Familie thematisiert. Eine Veränderung des jeweiligen Blickwinkels und der gewohnten Verhaltensmuster innerhalb der sozialen Kontexte wird auf diese Weise erst möglich.

\section{Systemisches Arbeiten mit Bezugsperson(en)} und Kind am Pferd Der Zeitpunkt, die Form und Dauer der Integration der Familien in die Arbeit mit dem Pferd erfolgt flexibel nach deren spezifischen Themen und Zielsetzungen. Im Nachfolgenden werde ich einige Aspekte dieser Arbeitsweise kurz vorstellen (Details hierzu in: Stockhausen 2009, 19f).

Innerhalb des Familiensystems sind die jeweiligen Strukturen und Beziehungsmuster weitgehend festgelegt. Wird nun mit dem Pferd ein „neues Mitglied“ in dieses Beziehungsgefüge aufgenommen, geraten die bisher bewährten Muster ins Wanken und machen somit die Neugestaltung der Familienstrukturen erforderlich.

Bereits beim Putzen des Pferdes werden die bisher etablierten Kommunikationsmuster und die jeweilige Verteilung der Rollen innerhalb der Familie sichtbar: „Wer verteilt die Aufgaben?“ oder „Wie selbstständig darf der Einzelne handeln?" und „Wie viel Nähe/Distanz braucht jeder/lässt der andere zu?". Eine ähnlich aufschlussreiche Aktivität ist das (gemeinsame) Führen des Pferdes. 
Der Umgang mit dem Pferd bietet ein "neutrales" Experimentierfeld, innerhalb dessen oftmals unbewusste Themen der Familie und des Einzelnen sowie unterschiedliche Beziehungskonstellationen erkennbar werden. Hier bieten sich zusätzlich viele Möglichkeiten, den eigenen Handlungsspielraum zu erweitern, sowie neue Wege im Umgang miteinander und in der Kommunikation zu erfahren und auszuprobieren.

Die Vorerfahrungen aus der Förderung des Kindes am Pferd kommen ihm in dem gemeinsamen Setting mit der Familie zugute, da es hier nicht länger die übliche Rolle des „Problemkindes“ innehat, sondern die Rolle des „Kompetenten“ erhält. Der Fokus der Eltern verändert und erweitert sich hier, er richtet sich nicht länger nur auf die Defizite des Kindes, sondern sie entdecken in ihm ungeahnte Stärken und Ressourcen.

\section{Die Autorin}

\section{Hildegard Stockhausen}

Dipl. Sozialpädagogin, Reitpädagogin (DKThR), Systemische Beraterin (SG), Ausbildung in klientenzentrierter Gesprächsführung und Psychomotorik, langjährige Erfahrung in der Heimerziehung und im Therapeutischen Reiten, seit

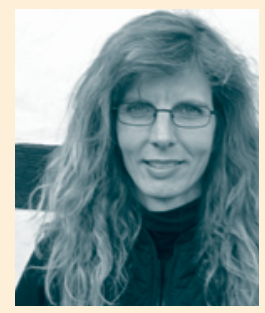
1996 eigene Praxis für Therapeutisches Reiten und Systemische Beratung, Referentin im Bereich der Heilpädagogischen Förderung mit dem Pferd unter psychomotorischen Aspekten sowie unter systemischen Gesichtspunkten

\section{Anschrift:}

\section{Hildegard Stockhausen}

Ruttscheiderstraße 28

53639 Königswinter

E-Mail:StockhausenHilde@aol.com
Auch das gemeinsame Reiten bietet viele neue Erfahrungsmöglichkeiten. Hier kommen vor allem die große körperliche Nähe (Intimität und Schutz) in Kombination mit der direkten Wahrnehmung des Anderen und der Erfahrung des gemeinsamen Anpassens an die Bewegungen des Pferdes zum Tragen. Das Gefühl von gemeinsamer Harmonie und körperlicher Nähe wird in der Regel als Ressource erfahren und als solche in den Alltag übertragen. Durch die zusätzliche Möglichkeit, unterschiedliche Sitzpositionen auf dem Pferd anzubieten, ergeben sich weitere Kontakt- und Kommunikationsmöglichkeiten (Details hierzu in: Stockhausen 2009, 19ff).

\section{Literatur}

Balgo, R., Voß, R. (1995): Kinder, die sich auffällig zeigen. Die systemisch-konstruktivistische Wende in der Psychomotorik. In: Kiphard, E. J., Olbrich, I. (Hrsg.): Psychomotorik und Familie. Psychomotorische Förderpraxis im Umfeld von Therapie und Pädagogik. Verlag Modernes Lernen, Dortmund, 167-194

Simon, F. B. (1993): Unterschiede, die Unterschiede machen. Klinische Epistemologie: Grundlagen einer systemischen Psychiatrie und Psychosomatik. 3. Aufl. Suhrkamp Verlag, Frankfurt am Main

Stockhausen, H. (2009): Integration systemischer Aspekte im Therapeutischen Reiten. Therapeutisches Reiten 36 (4), 16-21

Abstract: The article is about the foundations of systematic thinking and their integration into horse-assisted psycho-educational interventions. Some settings of this work are presented, e.g. the child centered horse-assisted intervention and the intervention with both, child and carer/parent are introduced.

Keywords: Systematic perspective, horse-assisted intervention, psycho-educational riding/ vaulting, relationship patterns, resourcing, joining, therapeutic riding 